\title{
Strobes: An Oscillatory Combustion
}

\author{
Justine M. L. Corbel, ${ }^{*}, \dagger+$ Joost N. J. Lingen, $^{\dagger}$ John F. Zevenbergen, ${ }^{\dagger}$ Onno L. J. Gijzeman, ${ }^{\ddagger}$ \\ and Andries Meijerink ${ }^{\ddagger}$ \\ ${ }^{\dagger}$ TNO Technical Sciences, P.O. Box 45, 2280 AA Rijswijk, The Netherlands \\ ${ }^{\ddagger}$ Condensed Matter and Interfaces, Debye Institute for Nanomaterials Science, Utrecht University, P.O. Box 80.000, 3508 TA \\ Utrecht, The Netherlands
}

ABSTRACT: Strobe compositions belong to the class of solid combustions. They are mixtures of powdered ingredients. When ignited, the combustion front evolves in an oscillatory fashion, and flashes of light are produced by intermittence. They have fascinated many scientists since their discovery at the beginning of the 20th century. However, the chemical and physical processes involved in this curious oscillatory combustion remain unknown. Several theories have been proposed: One claims that two different reactions occur: one during the slow dark phase and another during the fast flash phase. The alternation between the phases is ascribed to heat variations. Other theories suggest that the formation of intermediate species during the dark phase and the change of phase are caused by variations in their
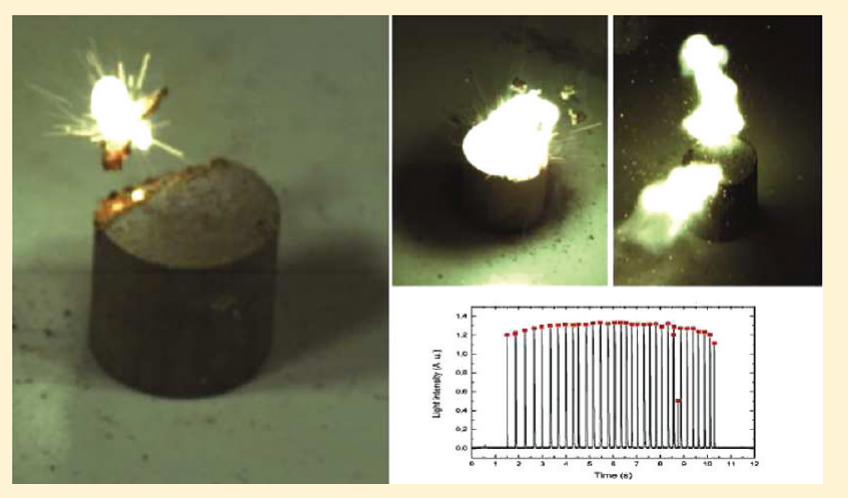
concentration. A ternary strobe composition with ammonium perchlorate, magnalium, and barium sulfate is analyzed. The role of barium sulfate is studied by replacing it by other metal sulfates that have different physical properties (melting points), and the burning of the compositions is recorded with a highspeed camera and a spectrometer coupled with a charge-coupled device (CCD) camera. Experimental results show noticeable differences in the physical and chemical processes involved in the strobe reactions.

\section{INTRODUCTION}

A strobe is a pyrotechnic composition that, when ignited, exhibits an oscillatory combustion. It produces flashes of white or colored light while burning. To qualify a good strobe, the flashes produced by a composition must be separated by sufficiently long and reproducible time intervals such that each flash can be individually observed. That implies that the flash frequency must be low enough, i.e., below $25 \mathrm{~Hz}$, and the transition between the phase before the flash, called the dark phase, and the flash phase must be sharp.

The strobe effect has applications in various fields, most notably in the fireworks industry and in the military area. In the latter case, it can be used for signaling when the frequency is well adjusted. ${ }^{1-4}$ The first reference to this kind of composition is found under the heading "Orion Flashing Guns" in an old mixing book of the firm Brocks Fireworks Ltd., published in $1898 .^{5}$ It was a mixture of sulfur, barium nitrate, magnesium powder, and aluminum pyroflake. Later on, other examples are mentioned in the literature. ${ }^{6-15}$

The discovery of new strobe mixtures is and has been the result of trial and error experimentation and is not based on understanding of strobing mechanisms. The first studies aimed at understanding the strobe mechanisms were carried out by Krone $^{16,1}$ and Wasmann ${ }^{17,18}$ in 1975. Krone experimented with compositions based on an alloy of aluminum and magnesium called magnalium, while Wasmann studied strobe compositions containing organic material and no magnalium. They both hypothesized that two different processes (physical and chemical) may occur during combustion: one during the dark phase and another during the flash phase. Another important study about strobes was undertaken by Shimizu. ${ }^{19}$ His hypothesis based on the work of Krone and Wasmann is described in Section II. However, several compositions were found that do not satisfy this hypothesis and still produce a strobe effect. ${ }^{20}$ At present, the understanding of the physical and chemical mechanisms governing a strobe reaction remains incomplete.

This paper aims to observe and analyze the role of some reactants of a classical strobe composition. The ternary strobe composition of ammonium perchlorate, magnalium, and barium sulfate is mentioned several times in the literature and has shown good strobing behavior. ${ }^{19,12,20}$ Therefore, this composition was chosen to act as the benchmark composition for the experiments described in this paper. Other compounds can be added in small amounts to improve the strobe performances (color of the flashes, frequency).

In this study, the role of the metal sulfate is analyzed. It has been claimed that a flash occurs when the metal sulfate reaches its melting point. ${ }^{21}$ To observe the influence of the melting point on the strobe behavior, barium sulfate was replaced by

Received: January 17, 2012

Revised: $\quad$ March 19, 2012

Published: April 9, 2012 


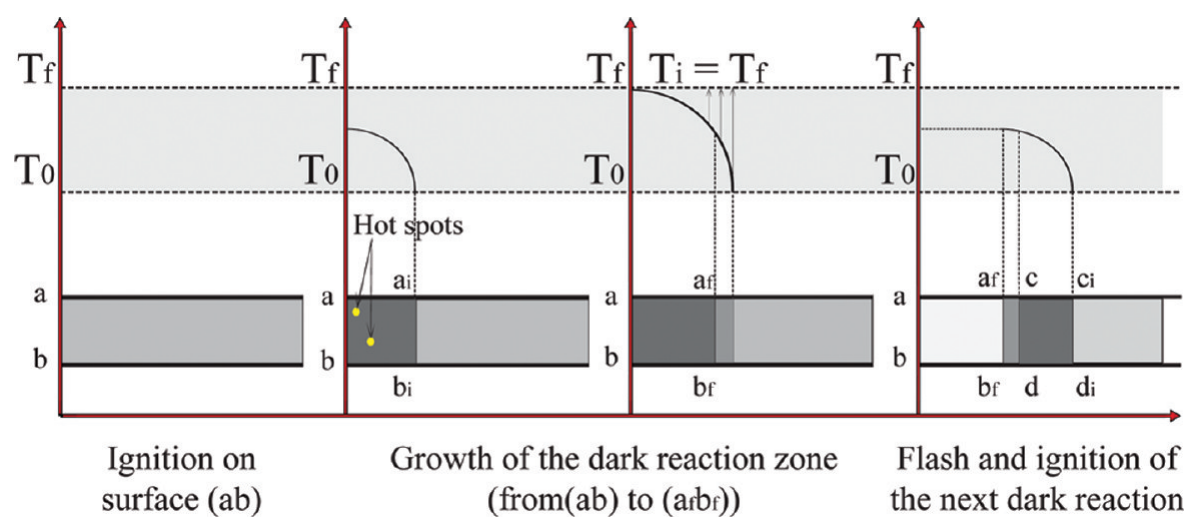

Figure 1. Scheme illustrating the Shimizu hypothesis about strobe mechanisms, adapted from ref 19 . The front flame propagates the left to the right. The evolution of the temperature is shown (vertical axis) as a function of time (horizontal axis); $T_{0}$ is the temperature at the beginning of the dark reaction. When the temperature reaches $T_{\mathrm{f}}$, the flash reaction is initiated. After the flash, the temperature drops, and the dark reaction in the next layer starts.

other metal sulfates (strontium, calcium, potassium, and sodium) that have a different melting point. The results were recorded with a charge-coupled device (CCD) camera coupled with a spectrometer and a high-speed camera.

\section{CURRENT KNOWLEDGE OF STROBE MECHANISMS}

The current common hypothesis used to explain strobes mechanisms was developed by Shimizu. ${ }^{19}$ After testing many strobe compositions, he argues that two reactions occur: a dark (or smolder) reaction and a flash (or light) reaction that alternate periodically. The trigger factor for the occurrence of a flash is the temperature of the reaction.

The mechanism is schematically shown in Figure 1. First the composition is ignited on the surface (ab), and the dark reaction starts. The temperature in the dark zone is not uniform, and hot spots appear on the surface, which grow as the temperature increases (as the heat accumulates). After a while, the maximum temperature of the dark zone reaches the ignition temperature of the flash composition, and the flash reaction starts. Therefore, a small layer of the dark zone $\left(a_{f} c b_{f}\right)$ remains on the burning surface because its temperature was too low to have been raised to the ignition temperature. This zone starts the next dark reaction leading up to the next flash reaction. In this manner, the dark and flash reactions periodically repeat.

This theory implies that strobe compositions contain two mixtures: one that gives rise to the dark reaction (the dark composition), and another that gives rise to the flash reaction (the flash composition). However, Jennings-White ${ }^{20}$ pointed out that several strobe compositions do not satisfy this criterion.

Two thermokinetic models have been developed. Both models are based on the strobe binary composition of magnesium and ammonium perchlorate. They both hypothesize that competing oxidation reactions occur between the three phases of magnesium (solid, liquid, and gaseous) with gas formed from the decomposition of ammonium perchlorate. The first model is a three-step model based on standard autocatalytic models. The model is described in Table $1 .^{22}$ This model is studied for $n=2$ and $m=3$, which corresponds to cubic autocatalysis. "23 "Cubic" stands for the form of the reaction rate, which is $r=a[\mathrm{~A}][\mathrm{B}]^{2}$ for the autocatalytic step of the model (Step 2 in Table 1 . This cubic autocatalysis process was originally used to model gas-phase reactions such as the oxidation of carbon monoxide. ${ }^{24}$ The two first steps of the
Table 1. Chemical Model Developed by Feng et al $^{22, a}$

\begin{tabular}{|l|l|l|}
\hline & \multicolumn{1}{|c|}{ chemical model } & simplified model \\
\hline step 1 & $3 \mathrm{Mg}_{(\mathrm{s}, 1)}+\mathrm{O}_{2} \rightarrow 2 \mathrm{MgO}+\mathrm{Mg}_{(\mathrm{g})}$ & $\mathrm{A}+\mathrm{B} \rightarrow \mathrm{B}+\mathrm{D}$ \\
\hline step 2 & $m \mathrm{Mg}_{(\mathrm{g})}+\mathrm{Mg}_{(\mathrm{s}, 1)}+\mathrm{O}_{2} \rightarrow n \mathrm{Mg}_{(\mathrm{g})}+2 \mathrm{MgO}$ & $\mathrm{A}+m \mathrm{~B} \rightarrow n \mathrm{~B}$ \\
\hline step 3 & $\mathrm{Mg}_{(\mathrm{g})} \rightarrow \mathrm{MgO}$ & $\mathrm{B} \rightarrow \mathrm{D}$ \\
\hline
\end{tabular}

model reflect the evaporation of magnesium and its partial oxidation; this corresponds to the dark phase. The last step of the model reflects the rapid oxidation of gaseous magnesium, which is the flash phase. The mass conservation gives rise to a nonlinear system of two equations. Steady-state solutions and oscillatory behavior were achieved using nonlinear analysis methods. The control parameter used was related to the diffusion coefficient for the diffusion between $\mathrm{Mg}_{(\mathrm{g})}$ and $\mathrm{O}_{2}$.

However, no temperature dependence is included in the model via the Arrhenius law, and a pool chemical approximation has been used for $\mathrm{Mg}_{(\mathrm{s}, 1)}$ (the concentration of this compound is assumed to be constant). Both observations are not consistent with a strobe reaction, but this model emphasizes the possible autocatalytic nature of the strobe reaction.

The second model created by Davies ${ }^{25}$ is inspired by the Sal'nikov model that was originally created to predict cool flames behavior, in particular the variation of temperature. ${ }^{26,27}$ The kinetic scheme is described as follows:

$$
\mathrm{P} \stackrel{k_{1}}{\rightarrow} \mathrm{X} \stackrel{k_{2}}{\rightarrow} \mathrm{B}+\mathrm{Q}_{2}
$$

A precursor $\mathrm{P}$, identified as $\mathrm{Mg}_{(\mathrm{s}, \mathrm{l})}$ is converted to a product $\mathrm{B}$, identified as $\mathrm{MgO}$, via an active intermediate $\mathrm{X}$, identified as $\mathrm{Mg}_{(\mathrm{g})} \cdot Q_{2}$ is the heat of reaction of the second reaction, and $k_{1}$ and $k_{2}$ are the rate constants of the first and the second reaction respectively. The following assumptions are made: The first reaction is supposed to produce no heat $\left(Q_{1}=0\right)$, and a chemical pool approximation is applied to precursor $\mathrm{P}$. Moreover, unlike the classical Sal'nikov model, both activation energies of the reactions are assumed to be positive and not only the second one.

The reactants identified as solid and liquid magnesium produce the intermediate species $\mathrm{X}$ (gaseous magnesium) in a first slow reaction. Those species are immediately consumed by the second reaction. This is the dark phase. Because of the 



Figure 2. (Left) Evolution of the temperature and the concentration of intermediate species with time during a strobe reaction. (Right) Phase plane plot where the temporal dependence is removed and the correlation between the temperature and the concentration of intermediate species is displayed (reproduced from Davies ${ }^{25}$ ).

exothermicity of the second reactions, the temperature increases, accelerating the consumption of $\mathrm{X}$ while its production remains constant. This is the flash phase. When there is no more intermediate species, the second reaction decelerates, the temperature decreases, and the production of $\mathrm{X}$ increases again. The cycle repeats itself.

The mass conservation equation applied to the intermediate $\mathrm{X}$ and energy balance equation give rise to a nonlinear system of two equations and four parameters (the ambient temperature, the initial concentration of reactants, the ratio of activation energies and the heat transfer coefficient $l$ ). As in the Sal'nikov scheme, the only bifurcation phenomena is the Hopf type that exhibits stable limit cycles. ${ }^{26}$ In other word, selfsustained oscillations can occur in the system described. The last parameter mentioned (the heat transfer coefficient $l$ ) is taken as the bifurcation parameter, and an oscillatory behavior is obtained when $l$ is between 0.5503 and 0.6777 . The left-hand graph of Figure 2 shows the evolution of the temperature and the concentration of intermediate species with time, while the right-hand graph shows the correlation between the two variables. Points $\mathrm{A}, \mathrm{B}, \mathrm{C}$, and $\mathrm{D}$ are characteristic states of the system during one cycle. Consequently, the flash occurs only if the heat generated is sufficient and if the concentration of "flash" species has reached a critical level to start the flash reaction. This implies that the dark and the flash reactions cannot be studied separately since the first one gives rise to the second one. The present understanding of strobe chemistry is not sufficient to corroborate any theory. The experiments described in this paper aim at providing new insights that can help explain the mechanisms governing strobe reactions.

\section{METHODS AND MATERIALS}

a. Experimental Section. Ten grams of each composition was prepared, including a binder solution composed of $10 \%$ nitrocellulose and $90 \%$ acetone. After mixing, the compositions were pressed (100 bar) into pellets of $1.5 \mathrm{~g}$ (including acetone) and then dried in a vacuum oven for $2 \mathrm{~h}$, giving a final weight of the pellet of about $1.26 \mathrm{~g}$. The diameter is $0.99 \mathrm{~cm}$, and the average height of the pellets is $0.917 \mathrm{~cm}$, so the average density is $0.446 \mathrm{~g} / \mathrm{cm}^{3}$. The pellet was placed on a metal plate and then ignited with a burner. All tests were performed in duplo.

b. Equipment. To capture the light emission of the strobes and to visualize the processes during the dark and the light reaction, high-speed camera images were recorded with a High-
Speed camera (Redlake digital imaging system, 5000 frames per second, resolution: $160 \times 160$ pixels). The emission spectra were recorded with a CCD camera (PIXIS System from Princeton Instruments, $1340 \times 100$ pixels $)$ coupled with a spectrometer (Princeton Instruments - Acton advanced SP2750A, $0.750 \mathrm{~m}$ focal length, imaging triple grating monochromator).

c. Compositions. The composition studied is a mixture of ammonium perchlorate, magnalium, and a metal sulfate (see Table 2). According to the theory described in Section II,

Table 2. Base Composition Used for the Experiment

\begin{tabular}{|c|c|c|c|}
\hline \multicolumn{4}{|c|}{ compositions (percentage by weight) } \\
\hline \multicolumn{3}{|c|}{ ammonium perchlorate } & 47.6 \\
\hline \multicolumn{3}{|c|}{ metal sulfate } & 13.5 \\
\hline \multicolumn{3}{|c|}{ magnalium } & 18.2 \\
\hline \multicolumn{3}{|c|}{ potassium dichromate } & 4 \\
\hline \multirow[t]{2}{*}{ binder } & nitrocellulose & 20 & 2 \\
\hline & acetone & & 18 \\
\hline total & & & \\
\hline
\end{tabular}

magnalium is used as fuel for both dark and flash reactions, and ammonium perchlorate and barium sulfate are the dark and the flash oxidizers, respectively. Potassium dichromate is added as a frequency modulator; it improves the sharpness and the regularity of the flashes. ${ }^{19}$ Finally, a solution of $10 \%$ nitrocellulose in acetone is added as a binder.

Metal sulfates were chosen because the metals themselves are in various forms commonly used in pyrotechnics. The metal sulfates used differ in melting point. The metal sulfates with their melting temperatures used are described in Table 3.

\section{Table 3. Metal Sulfates Used}

\begin{tabular}{lcc}
\multicolumn{1}{c}{ metal sulfates } & molecular formula & melting point \\
strontium sulfate & $\mathrm{SrSO}_{4}$ & $1879 \mathrm{~K}\left(1606^{\circ} \mathrm{C}\right)$ \\
barium sulfate & $\mathrm{BaSO}_{4}$ & $1853 \mathrm{~K}\left(1580^{\circ} \mathrm{C}\right)$ \\
calcium sulfate & $\mathrm{CaSO}_{4}$ & $1733 \mathrm{~K}\left(1460{ }^{\circ} \mathrm{C}\right)$ \\
potassium sulfate & $\mathrm{K}_{2} \mathrm{SO}_{4}$ & $1342 \mathrm{~K}\left(1069^{\circ} \mathrm{C}\right)$ \\
sodium sulfate & $\mathrm{Na}_{2} \mathrm{SO}_{4}$ & $1157 \mathrm{~K}\left(884^{\circ} \mathrm{C}\right)$
\end{tabular}





Figure 3. Collection of pictures showing one flash phase of the strontium sulfate composition.

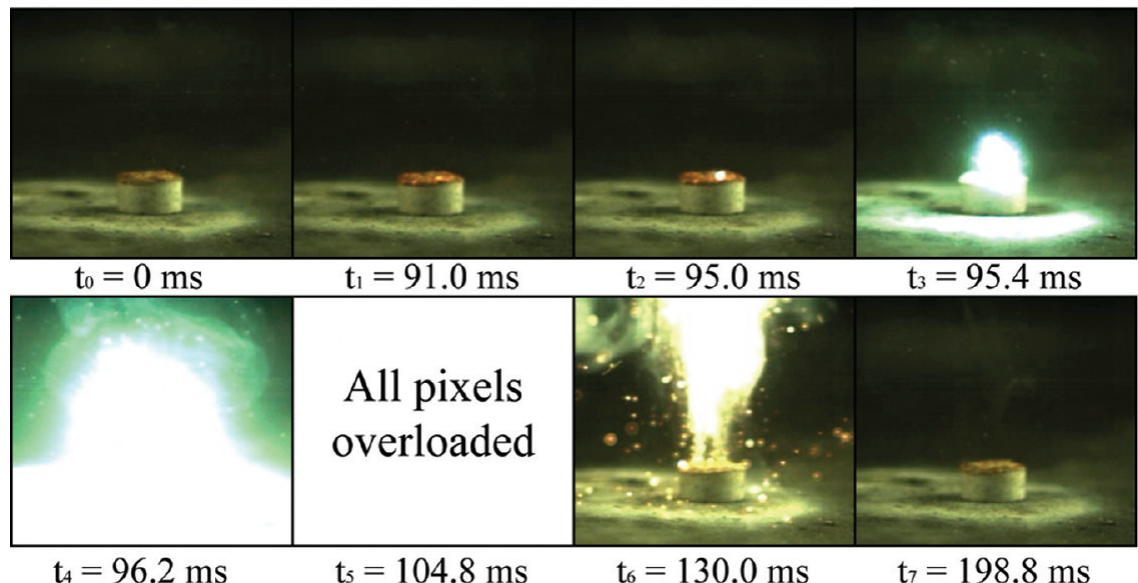

Figure 4. Collection of pictures showing one flash phase of the barium sulfate composition.
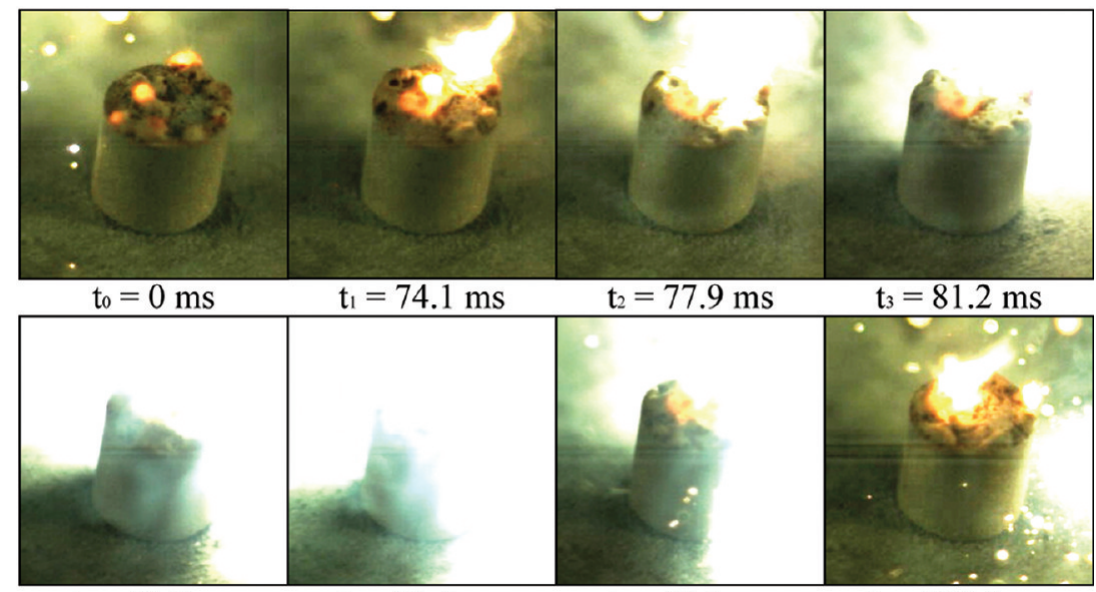

$\mathrm{t}_{4}=87.7 \mathrm{~ms}$

$\mathrm{t}_{5}=91.1 \mathrm{~ms}$

$\mathrm{t}_{6}=95.1 \mathrm{~ms}$

$\mathrm{t}_{7}=103.9 \mathrm{~ms}$

Figure 5. Collection of pictures showing one flash phase of the potassium sulfate composition.

\section{RESULTS AND DISCUSSION}

It has been claimed that a flash occurs when the metal sulfate reaches its melting point. ${ }^{21}$ The present experiments are aimed at investigating this effect systematically. The influence of the melting point of the sulfates used on the performance of the strobe (appearance of surface combustion, sharpness and regularity of the flashes) was studied using high-speed camera images. Also, comparisons between the combustion products present in the flash were made based on the emission spectra recorded.

a. Analysis of the High-Speed Camera Images. Figures 3 and 4 show typical images recorded during a flash for the strontium and barium sulfate compositions, respectively. Similar phenomena are observed. The cycle starts when the 

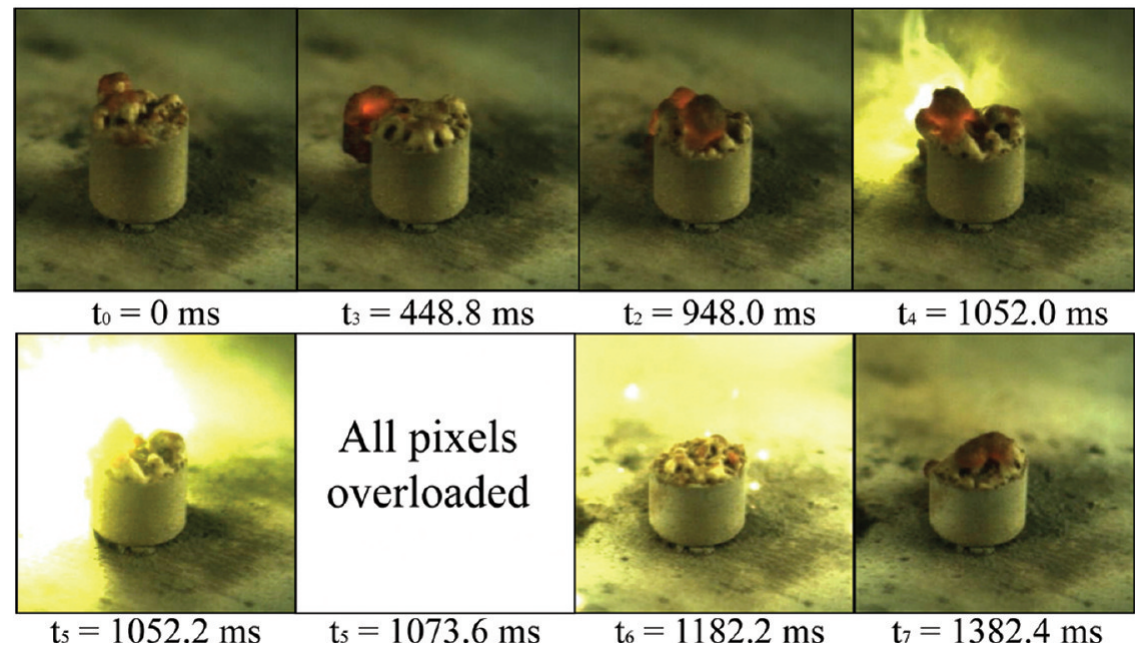

Figure 6. Collection of pictures showing one flash phase of the sodium sulfate composition.
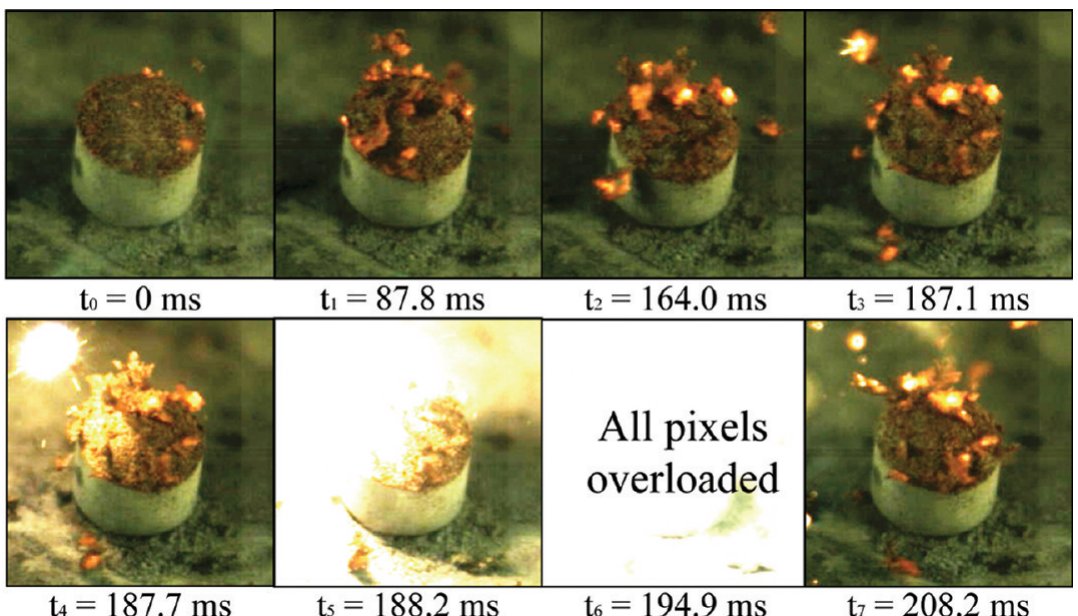

Figure 7. Collection of pictures showing one flash phase of the calcium sulfate composition.

pellet is ignited and a hot layer appears on top of the pellet. This phenomenon was also observed by Krone and Shimizu who called this layer the "semislag" and the "semireacted zone", $1,16,19$ respectively. Then, the layer propagates perpendicularly to the surface of the pellet and becomes thicker. The surface is heated by a reaction and it becomes red. Sometimes small parts of this layer are ejected from the pellet (see picture $t$ $=122.2 \mathrm{~ms}$ in Figure 3). Then, a short flash completely consumes the dark layer and initiates the next dark reaction. The pellet is consumed linearly, layer-by-layer. By contrast, the layers produced on top of the potassium- and sodium sulfatebased compositions (Figures 5 and 6) are composed of different zones, seemingly containing molten material, and sometimes foam appears at the surface (see picture $t=448.8$ $\mathrm{ms}$ in Figure 6). The color of the layers is not uniform, and their shapes are irregular; the top of the pellet is not consumed linearly, but small holes are created on the surface each time a flash occurs. For the sodium sulfate composition, the dark periods are longer, more material is consumed to create one dark layer, and less flashes are produced from one pellet, but they are more intense. The layer produced in the calcium sulfate composition is different from the other two groups of compositions (see Figure 7). It has a very irregular shape, and the pellet is not consumed linearly as in the case of the strontium and barium sulfate compositions, and many small flying particles are observed. However, the layer produced is more solid than that for the sodium and potassium sulfate compositions.

Moreover, during the dark phase, hot spots can be observed on the surface of the dark layer (see the picture $t=95.0 \mathrm{~ms}$ in Figure 4). They occur and grow while the dark reaction proceeds. Shimizu ${ }^{28}$ also observed this phenomenon. Those hot spots are the result of local heat accumulation in the dark layer, and they can evolve into small local flashes. Grose hypothesizes that hot spots occur on the molten part of the dark layer consuming those zones by a small flash. ${ }^{29}$ This indicates that the layer is partly molten in all compositions, and the flashes are probably initiated from those molten areas.

A general observation of the high-speed camera images is that the barium and the strontium sulfate compositions give rise to a good strobe behavior, while the other compositions produce very irregular and/or longer lasting flashes. This suggests that processes during the formation of the dark layer are important for strobe reactions. It must be uniform and cover the entire surface. Then, the layer is heated up homogeneously until it reaches a triggering point for the flash reaction. Subsequently, the flash occurs, and the next cycle starts. The presence of molten materials is important since the flashes and hot spots seem to be initiated from molten areas. However, if the compounds melt at too low temperatures, 


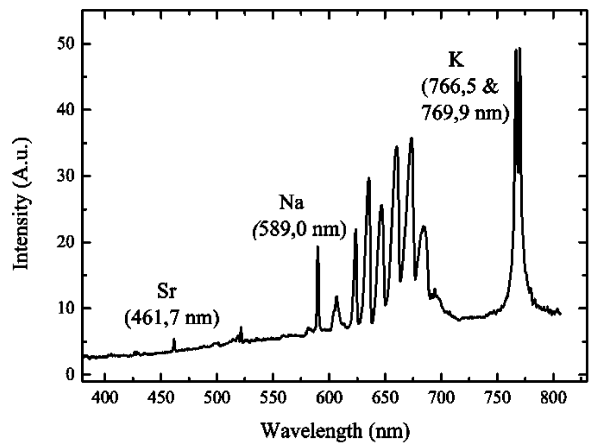

Spectrum 1:

from 380 to $800 \mathrm{~nm}$

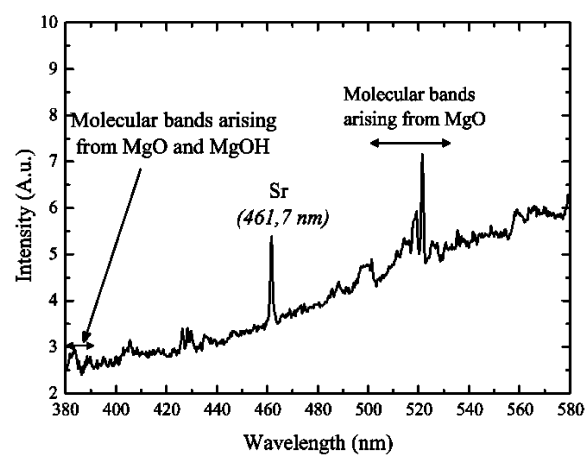

Spectrum 2:

from 380 to $580 \mathrm{~nm}$

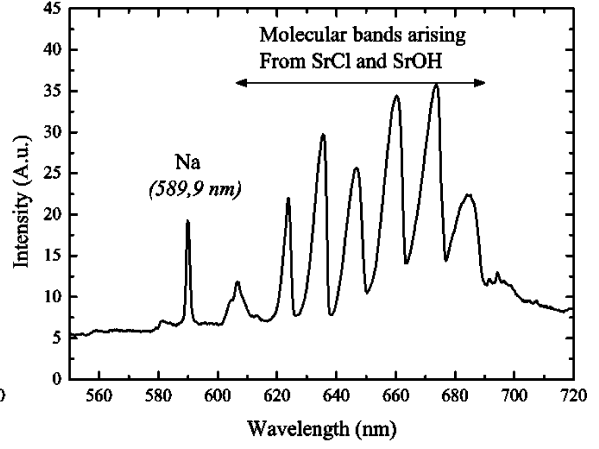

Spectrum 3:

from 560 to $720 \mathrm{~nm}$

Figure 8. Emission spectra recorded for the strontium sulfate composition.

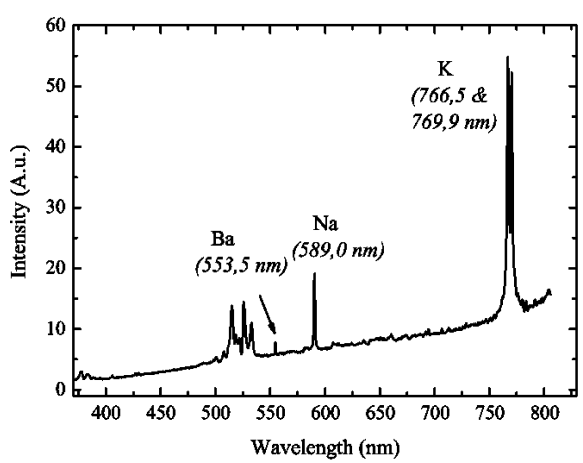

Spectrum 1: from 380 to $800 \mathrm{~nm}$

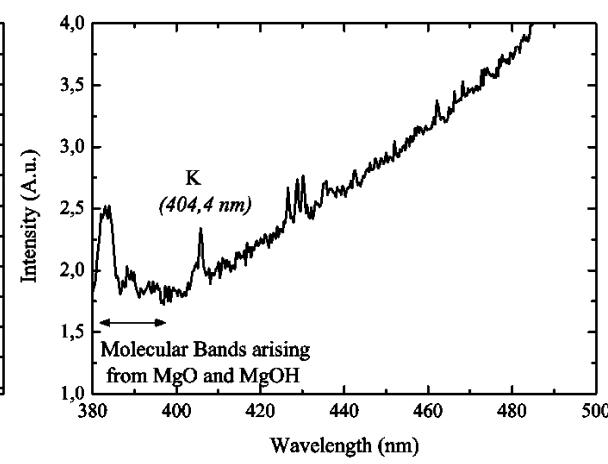

Spectrum 2: from 380 to $500 \mathrm{~nm}$

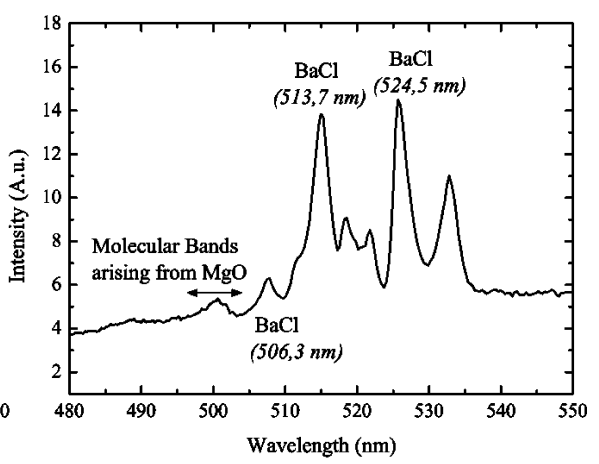

Spectrum 3: from 480 to $550 \mathrm{~nm}$

Figure 9. Emission spectra recorded for the barium sulfate composition.
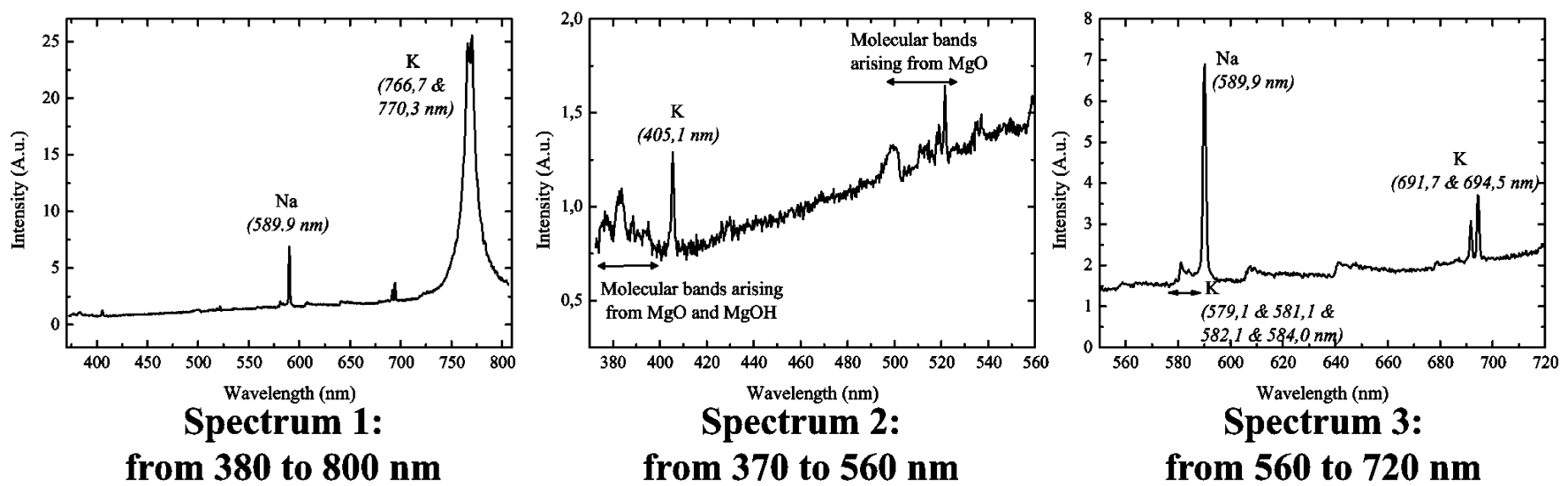

Figure 10. Emission spectra recorded for the potassium sulfate composition.

several flashes are initiated from the same layer, degrading the strobe effect, while too many hot spots occur, inhibiting a full flash. The layer formed is irregular and "moving" (formation of foam) when the gaseous species formed under the layer escape before the occurrence of a flash. The heat produced by the dark reaction is dissipated too quickly to ignite the flash reaction, and more material is necessary to accumulate enough heat for a full flash, resulting in a low flash frequency for, for instance, the sodium sulfate composition. On the other hand, the heat produced can also be distributed irregularly, producing many hot spots and weak successive flashes, as observed for the potassium sulfate composition (Figure 5).
To conclude, the barium and the strontium compositions produce a better strobe effect, which is attributed to the higher melting points of strontium and barium sulfates. It is important to maintain a solid dark layer favoring a homogeneous accumulation of heat and also of gaseous species that form under the surface. These two phenomena may be necessary for the occurrence of a flash. The temperature must be high enough to ignite the flash, but a specific concentration of gaseous species may also be essential to the manifestation of sharp flashes. The different behaviors observed (sharpness and regularity of flashes, gaseous species) are investigated further using the $\mathrm{CCD}$ camera and the spectrometer. 

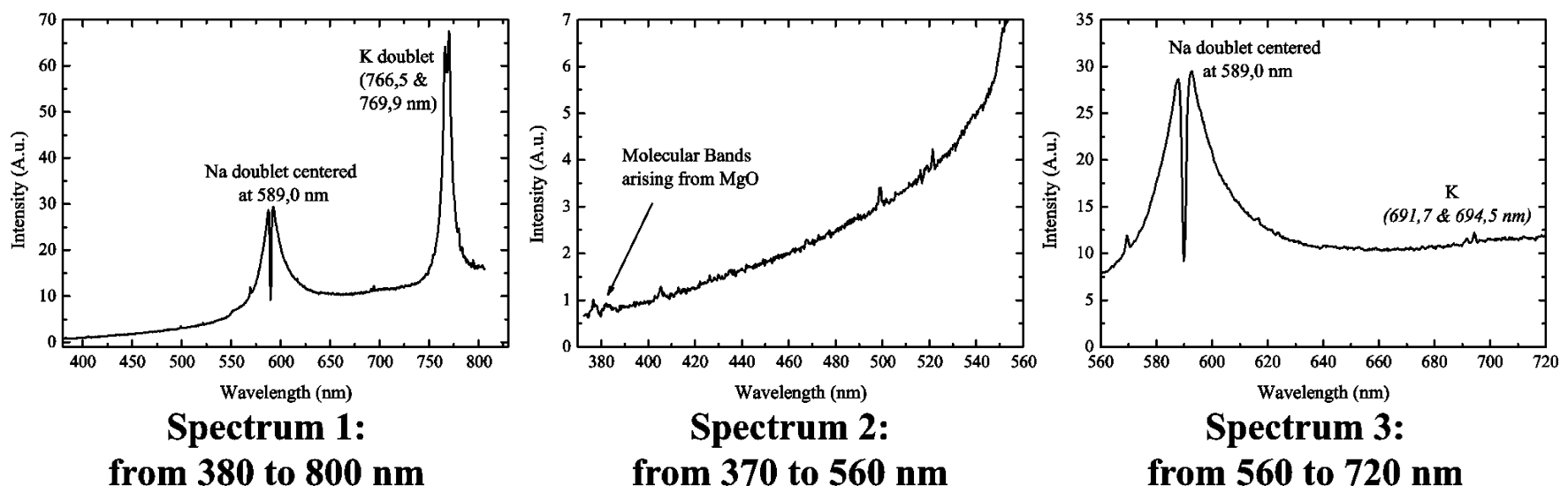

Figure 11. Emission spectra recorded for the sodium sulfate composition.
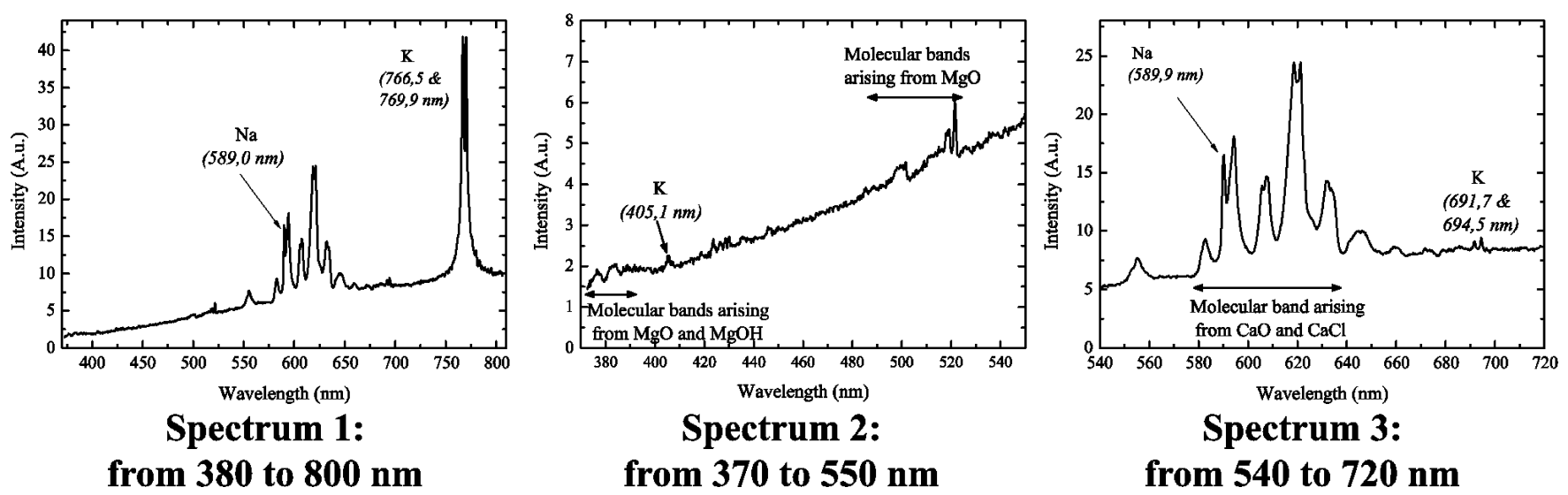

Figure 12. Emission spectra recorded for the calcium sulfate composition.

b. Analysis of the Spectra. Emission spectra were recorded for all the compositions in the visible range 370$810 \mathrm{~nm}$ (see the first spectrum in Figures 8-12). After which high resolution spectra were recorded of the relevant zone of all the spectra (see spectra 2 and 3 in each figure). They were analyzed with the help of databases of emission spectra of a variety of atomic and molecular gaseous species. ${ }^{30,31}$

The sodium line emission at $589 \mathrm{~nm}$ is present in all the spectra as an unresolved doublet, which is unsurprisingly particularly visible in the spectrum of the sodium sulfate composition (Figure 11). Except for this composition, this spectral line is the result of a sodium contamination present in the ingredients used. The potassium doublet centered at 767 $\mathrm{nm}$ also appears in most of the spectra for the same reason. Furthermore, a weak spectral line appears in some spectra at $405 \mathrm{~nm}$, which may be the result of an emission from potassium. Note that the line is most clearly visible for the sample with potassium sulfate. The source for potassium is potassium dichromate. Its decomposition may provide the potassium atoms responsible for those emission lines. Further analysis of spectrum 2 of all the figures shows molecular bands that can be assigned to vibronic emission lines of magnesium hydroxide and magnesium oxide from 370 to $390 \mathrm{~nm}$ and from 495 to $500 \mathrm{~nm}$, respectively (maxima at $497.3,498.3,499.5$, and $500.6 \mathrm{~nm}$ ). Those bands appear in all the spectra with different intensities. They may be quenched by other spectral lines such as in Figure 7 where the sodium lines are so intense that the other lines and bands are barely noticeable.
The spectrum of the strontium sulfate-based composition (see Figure 8) shows spectral lines that arise from the excitation of the atoms of strontium at $461.7 \mathrm{~nm}$ and molecular bands due to vibronic transitions of strontium monochloride and strontium hydroxide (peaking at 623.9, 635.9, and $673.9 \mathrm{~nm}$ ). Other bands appear at $606.3,616.1$, and $684.6 \mathrm{~nm}$. They have not been fully identified, but they are not present in the other spectra. Consequently, they may be assigned to vibronic transitions of a molecule containing strontium. The barium sulfate composition spectra also contain two molecular bands that are assigned to vibronic transitions of barium monochloride at 513.7 and $524.5 \mathrm{~nm}$ (see Figure 9). The spectral line present at $553.5 \mathrm{~nm}$ is consistent with the emission from the barium atom. Other molecular vibronic bands are present (peaking at 506.3, 516.8, 520.4, 531.6 nm). They may be the result of the vibronic transitions for barium monochloride, barium oxide, or barium hydroxide. The emission spectra of the calcium sulfate composition (see Figure 12) also reveal the presence of calcium monochloride and calcium oxide in the flame (molecular bands at 593.7, 618.7, and $621.2 \mathrm{~nm}$ for calcium monochloride and maxima at 605.7 and $607.3 \mathrm{~nm}$ for calcium oxide). By contrast, the spectra of the compositions containing potassium and sodium sulfate are dominated by atomic emission lines from sodium and potassium (see Figures 10 and 11). Evidently, the emission from sodium and potassium is more efficient than that of the molecular species, which also leads to the observation of strong sodium emissions for compositions in which sodium is present as an unintentional contamination. 


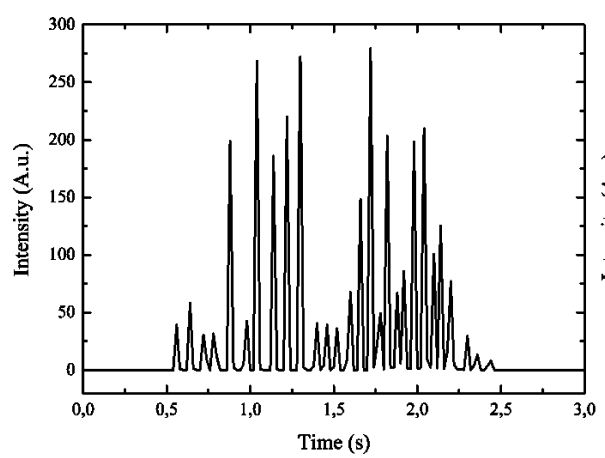

Strontium sulfate composition Average frequency: $13.5 \mathrm{~Hz}$

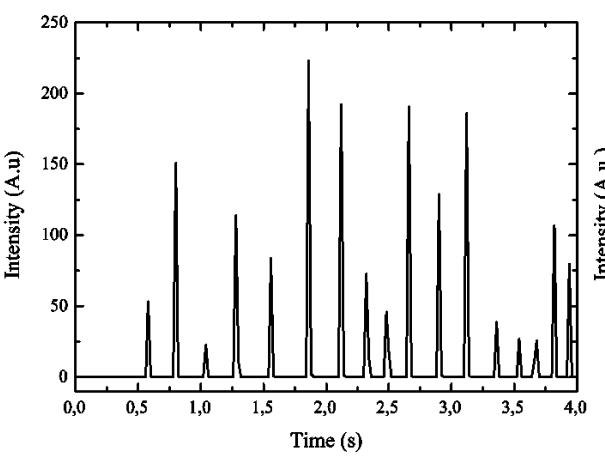

Barium sulfate composition Average frequency: $5.14 \mathrm{~Hz}$

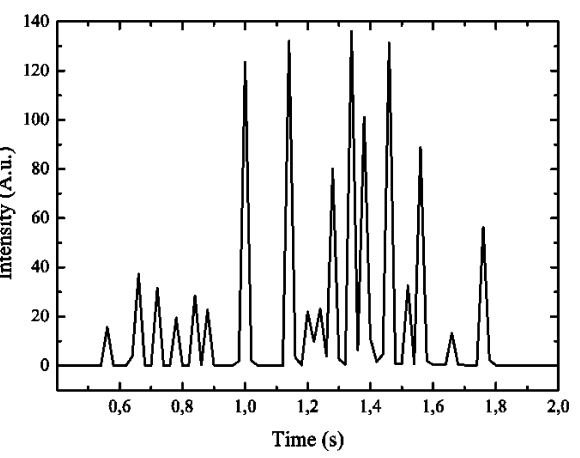

Calium sulfate composition Average frequency: $11.12 \mathrm{~Hz}$
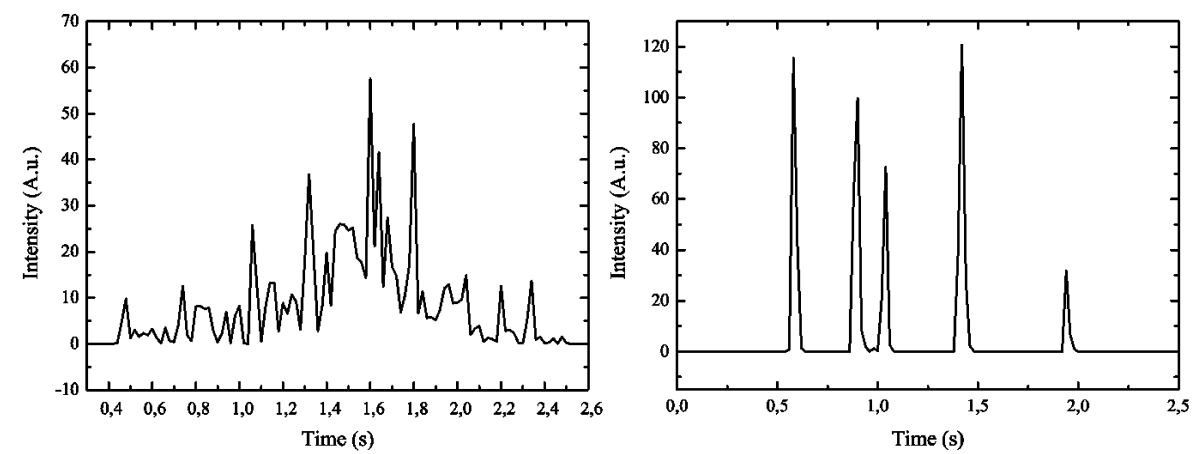

Potassium sulfate composition
Average frequency: $11.93 \mathrm{~Hz}$

Sodium sulfate composition Average frequency: $2.75 \mathrm{~Hz}$

Figure 13. Intensity evolution versus time for all compositions tested.

All the spectra show the presence of magnesium oxide and magnesium hydroxide in the combustion products. The presence of those bands imply the melting and/or evaporation of magnalium (melting point: $460{ }^{\circ} \mathrm{C}^{32}$ ) and the oxidation of magnesium. Then the burning of the barium, strontium, and calcium compositions gives rise to metal monochloride and oxide. It suggests that those metal sulfates decompose during the process. On the other hand, only atomic lines appear in the spectra for the sodium and the potassium compositions. This could mean that sodium monochlorides and oxides are not produced by the reaction, or maybe they are produced but not efficiently excited during the flash process. However, the mechanisms involved in those two groups of compositions seem different from the other compositions. This feature can also be observed with analysis of the high-speed camera images.

In addition, the CCD camera records the temporal evolution of the emission spectra. Thus, the variation of the intensity of any relevant spectral line can be used to record the frequency of flashes and also evaluate the sharpness of the flashes. The resulting graphs are given in Figure 13. The barium- and strontium sulfate-based compositions have regular and sharp flashes in comparison to the other compositions. The sodium and calcium sulfate compositions also have sharp flashes but less regular and with a lower frequency for the sodium composition. The potassium sulfate-based composition produces very irregular and indistinguishable flashes.

To conclude, the results obtained from the spectrometer corroborate the high-speed camera images: barium- and strontium sulfate-based compositions show the best strobe behavior, and their sulfate ingredients also have higher melting points. So the conclusion might be drawn that a high melting point is crucial for a regular strobe effect.

\section{CONCLUDING REMARKS}

Previous studies on strobe reaction emphasize the importance of thermal and chemical parameters in the occurrence of flashes. The influence of sulfates on a classical strobe composition was studied: five metal sulfates (barium sulfate, strontium sulfate, calcium sulfate, potassium sulfate, and sodium sulfate) were tested in combination with ammonium perchlorate and magnalium. The sulfates all have different melting points. The results of time-resolved emission spectra (CCD/spectrometer) and high-speed camera images (high speed camera) of the ignited strobe compositions reveal that the compositions containing sulfates with higher melting points (strontium/barium sulfate compositions) give the best strobe effect.

Three groups of compositions were identified from the results: the high melting point metal sulfate compositions that include the strontium and the barium-based compositions, the low melting point metal sulfate compositions with sodium and potassium sulfates, and intermediate melting point metal sulfate compositions, for instance, calcium sulfate. The spectra of the first group (high melting point metal sulfate) reveal in both cases the presence of metal monochloride, oxide, and hydroxide in the gas excited during the flash phase. This implies that the sulfates decompose during the process, and then the metal is oxidized or forms a monochloride. In addition, the high-speed camera images show the formation of a uniform and partly solid layer on top of the pellet. This layer is heated up almost 
uniformly, and flashes seem to be initiated from the molten areas present on the surface. The resulting strobe effect is characterized by sharp flashes at regular time intervals.

To the contrary, the strobe effect observed for the composition of the second group (low melting point metal sulfate) is not as good; the high-speed camera images show the formation of an irregular layer that is almost liquid, and that leads to irregular and longer lasting flashes. In the emission spectra recorded, only atomic peaks are observed during the flash phase. This may imply that the sulfate decomposes, but the metal does not react with oxygen and monochloride. However, the most probable hypothesis is that either the temperature is not high enough to excite those molecular species efficiently and the emission intensity is too low to observe the vibronic emission lines next to the very efficient sodium and potassium emission lines, or the molecular species formed during the process do not emit in the visible but in another range such as the microwave range line, as, for instance, with sodium monochloride. ${ }^{33}$ Finally, the strobe effect produced by the third group of compositions is an intermediate state between the good strobe produced by the first group of compositions and the erratic strobe effect of the second group. The spectra show the presence of metal monochloride, oxide, and hydroxide during the flash phase as observed for the first group. Consequently, the sulfate also decomposes during the process. However, the layer formed during the dark phase shows hot spots and irregular flashes, just like the second group, except that the top layer seems more solid.

The results imply that metal sulfates participate in the dark reaction and that their melting points are important in the formation of a homogeneous dark layer. Compositions that contain metal sulfates with a high melting point produce a better strobe effect. Metal sulfates with low melting points seem to prevent strobe reactions with regular intervals and sharp flashes. Because of the melting of the sulfate in the layer, the full surface cannot be ignited. Still, the mechanism and processes responsible for strobe reactions remain largely unknown. Further studies are necessary to provide a better understanding and to elucidate the mechanism behind the fascinating strobe reactions.

\section{AUTHOR INFORMATION}

\section{Corresponding Author}

*E-mail: justine.corbel@tno.nl; phone: +31888661146.

\section{Notes}

The authors declare no competing financial interest.

\section{ACKNOWLEDGMENTS}

Grateful acknowledgment is given to the TNO Defense Ph.D. Fund for sponsoring this opportunity of working on strobe compositions, to the TNO Defense Security and Safety for providing the equipment used for this study, and to our supervisors for their support and their useful suggestions and comments.

\section{REFERENCES}

(1) Krone U. Pyrotechnischer satz sur Strablungsemission, 1973, Patent no.

(2) Cornia R. P. Composition for producing flickering signals, 1976, Patent no.

(3) Donoho D. W. Composition for pulsating flares, 1982, Patent no.

(4) Sayles D. C. Signal or rescue flare of variable luminosity, 1989, Patent no.
(5) Brock, A. S. Pyrotechnics: The History and Art of Fireworks Making; Daniel O'Connor: London, 1922.

(6) Sidirov A. I., Kravchenko I. P., Antonov V. M., Arsh M. M., Madyakin F. P., Godoshnikova L. Y., Ugd'kova A. S. Pyrotechnics composition for yellow flame, 1963, Patent no.

(7) Sidorov A. I., Kravchenko I. P., Antonov V. M., Kovakenko P. V., Gorovoi V. R. Pyrotechnic composition for green flames, 1969, Patent no.

(8) Sidorov A. I., Kravchenko I. P., Antonov V. M., Silin N. A., Kovalenko P. V., Arsh M. M., Madyakin F. P., Baturova G. S., Gorovoi V. R. Pyrotechnical composition, 1969, Patent no.

(9) Winokur, R. Pyrotechnica 1978, II, 28-35.

(10) Cardwell, R. G. Pyrotechnica 1979, V, 6-24.

(11) Shimizu, T. Pyrotechnica 1985, $X, 5-32$.

(12) Meinhart, J. Pyrotechnica 1985, XI, 2-4.

(13) Jennings-White, C. J. Pyrotech. 2004, 20, 7-16.

(14) McCaskie, E. Pyrotechnica 1993, XV, 35-45.

(15) Hiskey M. A., Chavez D. E. High-nitrogen energetic material based on pyrotechnic compositions. International Patent No. WO 98/ 54113, 1998.

(16) Krone, U. Pyrotech.: Grundlagen, Technol. Anwend. 1975, 225237.

(17) Wasmann, F. Pyrotech.: Grundlagen, Technol. Anwend. 1975, 239-250.

(18) Wasmann, F. Proc. Int. Pyrotech. Semin. 1976, 5th, 643-651.

(19) Shimizu, T. Pyrotechnica 1982, VIII, 5-28.

(20) Jennings-White, C. Pyrotechnica 1992, XIV, 33-45.

(21) Haarmann D. Pyrotechnic Guild International, Bulletin No. 46, 1985, pp 8-13.

(22) Feng, C.; Zeng, Q.; Du, Z. J. Chem. Soc., Faraday Trans. 1996, 92, 2971-2975.

(23) Gray, P; Scott, S. K Chem. Eng. Sci. 1984, 39, 1087-1097.

(24) Gray, B. F.; Roberts, M. J. Proc. R. Soc. London, Ser. A: Math., Phys. Eng. Sci. 1988, 416, 403-424.

(25) Davies, M. L. J. Pyrotech. 2008, 27, 42-49.

(26) Gray, B. F.; Roberts, M. J. Proc. R. Soc. London, Ser. A: Math., Phys. Eng. Sci. 1988, 416, 425-441.

(27) Lignola, P. G.; Reverchon, E. Prog. Energy Combust. Sci. 1987, $13,75-96$.

(28) Shimizu, T. Proc. Int. Pyrotech. Semin. 1991, 16, 47-53.

(29) Grose, R. I.; Cartwright, M.; Bailey, A. J. Pyrotech. 1996, 4, 1-4.

(30) Ralchenko Y., Kramida A. E., Reader J. NIST Atomic Spectral Database, 2008.

(31) Pearse, R. W. B., Gaydon, A. G. The Identification of Molecular Spectra; Chapman and Hall: Cambridge, U.K., 1976.

(32) Kosanke, $\mathrm{K}$ et al. Pyrotechnic Chemistry; Number 4 in the Pyrotechnic Reference Series; Journal of Pyrotechnics, Inc: Whitewater, CO, 2004.

(33) Lovas F. J., Tiemann E., Coursey J. S., Kotochigova S. A., Chang J., Olsen K., Dragoset R. A. NIST Diatomic Spectra Database, 2009. 